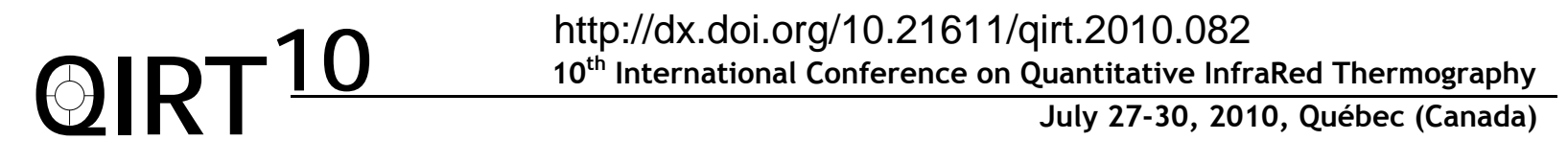

\title{
Free carrier diffusion-wave modulation of a sub-bandgap cw laser beam
}

by A. Mandelis and J. Tolev

Center for Advanced Diffusion-Wave Technologies (CADIFT), Mechanical \& Industrial Engineering, University Toronto, 5 King's College Road, Toronto, ON M5S 3G8, Canada

Nowadays the use of excited free carriers in semiconductors for light modulation and switching is becoming increasingly popular because of speed, absence of moving components, negligible parasitic heating, no effects from external fields and no electrical, thermal or acoustic influences on the modulating/switching active area. In this work we describe the modulation of an unmodulated (cw) 1550-nm sub-bandgap laser beam with power $P_{c w}$, by the spatially dependent absorption coefficient of free carriers created in semiconductor medium following absorption of a super-bandgap laser radiation. The semiconductor medium were one and two-side polished Silicon wafers. As a super-bandgap radiation were used two collinear laser beams, one harmonically modulated (ac) at angular frequency $\omega$ with power $P_{a c}(t)$ and other unmodulated (dc) with power $P_{d c}$, both with different combinations of the wavelengths $355 \mathrm{~nm}, 830 \mathrm{~nm}$, and $980 \mathrm{~nm}$. When the super-bandgap beams $P_{a c}(t)$ and $P_{d c}$, are off the sub-bandgap laser absorption coefficient is due to residual (background) absorption and has a small value: $a_{c w}=a_{0}$. When $P_{a c}(t)$ and $P_{d c}$, are on, their radiation is strongly absorbed within a thin surface layer of the material and inter-band photocarrier excitation occurs. The time-dependent absorption coefficient of the sub-bandgap radiation can be expressed as:

$$
\alpha_{c w}(z, t)=\alpha_{0}+\alpha_{f c}(z)+\frac{1}{2} \alpha_{f c}(z, \omega)\left(1+e^{i \omega t}\right)
$$

Here $a_{f c}$ is the free-carrier absorption coefficient at the sub-bandgap beam wavelength and it is function of the excess free carrier density $\Delta N(z, \omega)$ generated by the $(d c)$ and $(a c)$ superband-gap laser beams. The subband-gap laser radiation absorptance, $A_{c w}(\omega, t)$, defined as the absorption coefficient multiplied by the total thickness $L$ of the semiconductor, can be written as:

$$
\begin{aligned}
A_{c w}(\omega, t)=\alpha_{0} L+\int_{0}^{L} \alpha_{f c}(z) d z & +\frac{1}{2}\left(1+e^{i \omega t}\right) \int_{0}^{L} \alpha_{f c}(z, \omega) d z= \\
& =\alpha_{0} L+C\left(\int_{0}^{L} \Delta N(z) d z+\frac{1}{2}\left(1+e^{i \omega t}\right) \int_{0}^{L} \Delta N(z, \omega) d z\right)
\end{aligned}
$$

where $C$ is a sub-bandgap laser radiation wavelength depending constant.

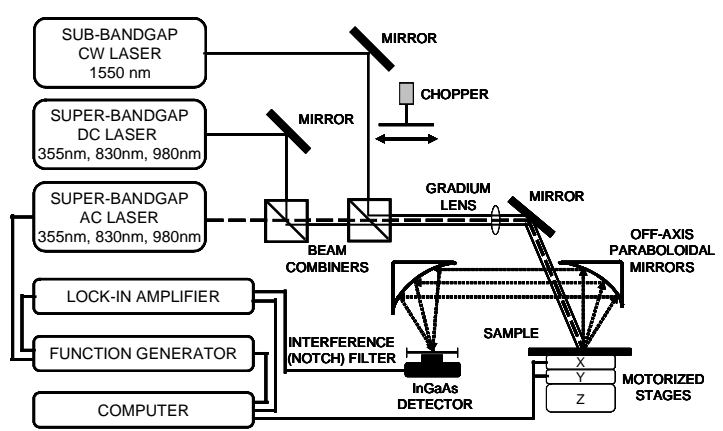

a

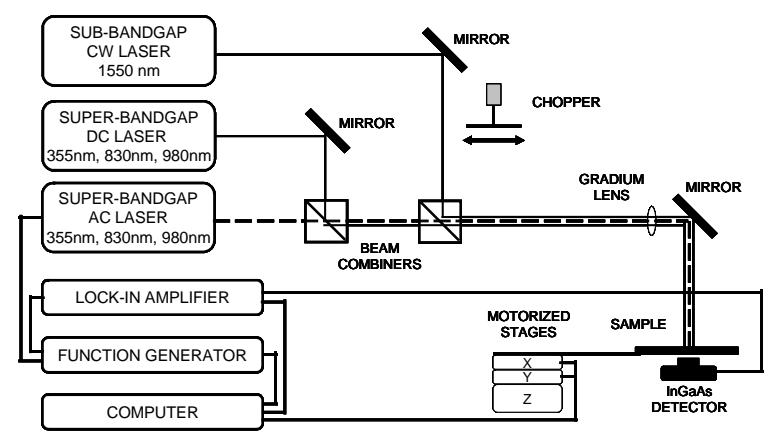

b

Fig. 1. Experimental setups. (a) reflected and (b) transmitted sub-bandgap ac power $P_{c w}(\omega, t)$.

Modulated sub-bandgap radiation $P_{c w}(\omega, t)$ is generated by the time-dependent absorptance $A_{c w}(\omega, t)$. If neglect the infinite inter-reflections of the sub-bandgap laser radiation from the semiconductor top and bottom surfaces it can be shown that: 


$$
\left.P_{c w}(\omega, t)=P_{c w} T_{0} e^{-\left(\alpha_{0} L+C T_{1} \int_{0}^{L} \Delta N(z) d z\right.}\right)\left[1-\frac{1}{2} m(\omega)\left(1+e^{i \omega t}\right)\right]
$$

where:

$$
m(\omega) \equiv C T_{2} \int_{0}^{L} \Delta N(z, \omega) d z
$$

Here $m(\omega)$ is the modulation depth of the optoelectronic modulator and $T_{0}, T_{1}$ and $T_{2}$ are terms involving the absorption, reflection and transmission coefficient of the semiconductor at the wavelength of the subband-gap laser.

Two groups of experiments were performed on the experimental setups presented on Figure 1 . In the first group the free carrier generated sub-bandgap ac power $P_{c w}(\omega, t)$ was reflected from the rough back surface of the one-side polished wafer. To minimize the contribution from synchronously emitted photo-carrier radiometric (PCR) power contributions, $P_{P C R}(\omega, t)$, as well as from back scattered sources power, $P_{a c}(t)$ and $P_{d c}$, to the modulated back propagated sub-bandgap power, an interference filter was positioned in front of the InGaAs photodetector, transmitting the $1550 \mathrm{~nm}$ radiation only. In the second group of experiments, $P_{c w}(\omega, t)$ was transmitted through the two-side polished wafer and the transmitted modulated sub-bandgap power was monitored. In this case any $P_{a c}(t)$ and $P_{d c}$, were eliminated through absorption near the top (front) surface of the wafer. The presence of PCR signal power was negligible. In the both groups of experiments the photodetector signal was proportional to $m(\omega)$, Eq. (4).
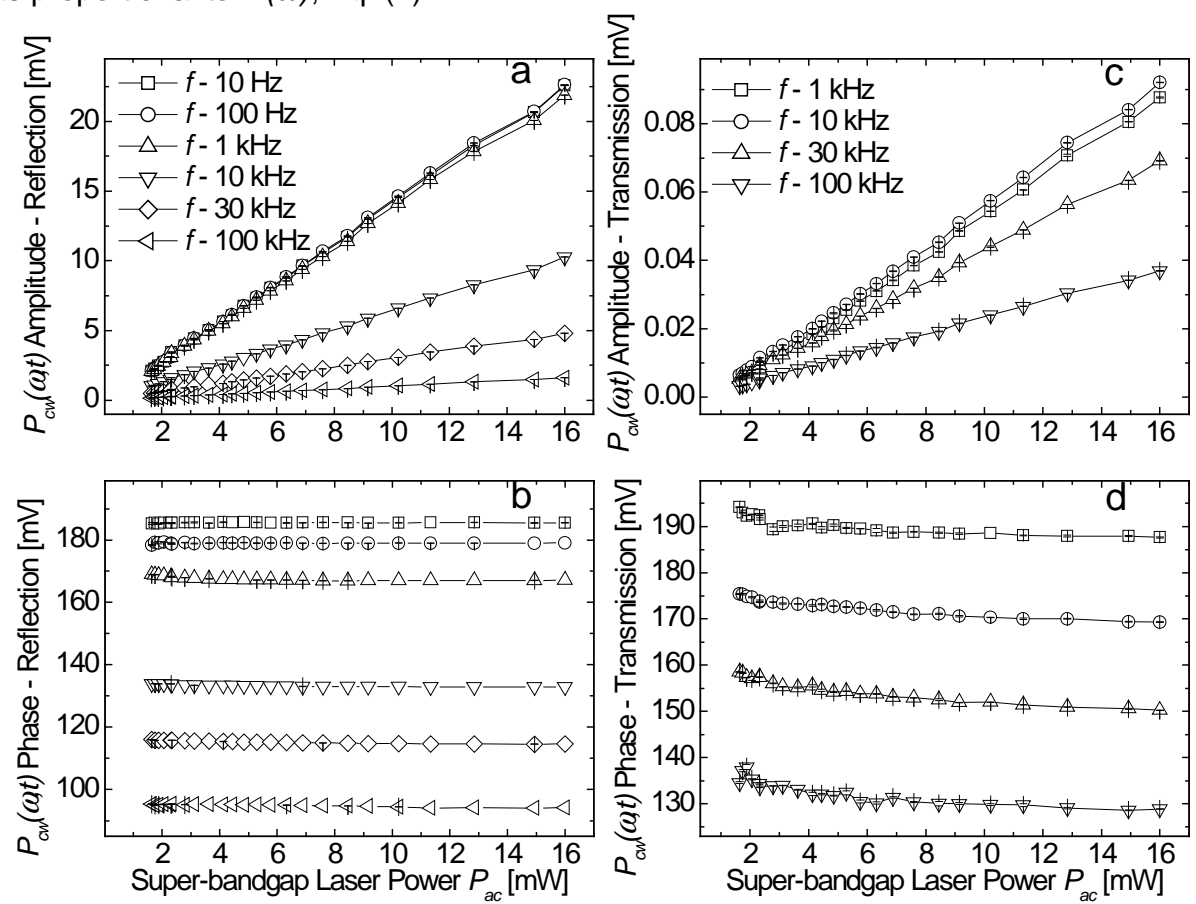

Fig. 2. Free-carrier modulation super-bandgap laser power $P_{a c}(t)$ light scan; $P_{d c}=0$. (a) and (b) amplitude and phase-reflection (back-propagation) modulation; (c) and (d) amplitude and phase-transmission modulation.

To determine the reference value for the optoelectronic modulator, the modulated signal corresponding to $m(\omega)=1$ or $100 \%$ modulation of $P_{c w}$, the super-bandgap laser radiations $P_{a c}(t)$ and $P_{d c}$, were turned off, and $P_{c w}$ was modulated with a mechanical chopper at $1 \mathrm{kHz}$. These reference measurements were made in both groups of experiments without changing the relative positions of the photodetector, wafer and $P_{c w}$ laser beam. Figures $\mathbf{2} \mathbf{a}$ and $\mathbf{2} \mathbf{b}$ show the amplitude and phase of back propagated $P_{c w}(\omega, t)$ from the rough back surface of one-side polished wafer for six different frequencies. The freecarrier modulation depth of $P_{c w}(\omega, t)$ obtained using $P_{a c}(t)$ at $16 \mathrm{~mW}$ and $P_{d c}=0$ at $1 \mathrm{kHz}$ relative to $100 \%$ modulation of the sub-bandgap beam by the mechanical chopper was found to be $3.4 \%$. Figures $\mathbf{2 c}$ and $\mathbf{2} \mathbf{d}$ show the amplitude and phase of transmitted $P_{c w}(\omega, t)$ through the two-side polished wafer for four different frequencies. Using the same modulation depth reference procedure as for one side polished wafer, it was found that the modulation depth of $P_{c w}$ was only $0.36 \%$.

Based on the presented theoretical and experimental evidence, there are excellent prospects for further improvement of this optical driven modulation method to produce modulating/switching devices for use with the modern photonic technologies. 